MATEC Web of Conferences 13, 05007 (2014)

DOI: $10.1051 /$ matecconf/ 20141305007

(C) Owned by the authors, published by EDP Sciences, 2014

\title{
Development of Reliability Analysis Toolkit for Analysing Plant Maintenance Data
}

\author{
Hilmi Hussin ${ }^{1, a}$, Ainul Akmar Mokhtar ${ }^{1}$, Masdi Muhammad ${ }^{1}$ and Mohd Amin Abdul Karim ${ }^{1}$ \\ ${ }^{1}$ Mechanical Engineering Dept., Universiti Teknologi PETRONAS, 31750, Perak, Malaysia
}

\begin{abstract}
Plant failure and maintenance data can be found in abundance, however, their utilization as a basis for improvement action is not fully optimized. This happens because many reliability analyses based on plant data are tedious and time consuming due to nonstandardized nature of the data being recorded. To overcome this issue, this study aims to develop a computer based reliability analysis toolkit to facilitate proper analysis of plant data. The toolkit can be used to perform both exploratory and inferential analysis. The developed toolkit has been demonstrated capable of assisting data gathering and analysis as well producing estimation of reliability measures.
\end{abstract}

\section{Introduction}

Plant maintenance and failure data are important input for effective decision making in improving plant performance. These data can be in abundance in plant and contain details about equipment and system downtime history, types, time, duration and causes. Nevertheless, their utilization as prime data source for driving improvement is not fully optimized due to several reasons. First, these data commonly exist in various locations, types and forms. They are not stored in a common database, but recorded separately at different places i.e. engineering, maintenance, production and computerized maintenance management system (CMMS). Some data are found recorded in unsystematic and nonstandardized format. Hence, due to these, data gathering becomes very time consuming task which can easily discourage engineer from performing effective analysis on plant data. Second, there is still lacks of knowledge among engineers on proper method of analyzing such data. Even though, there exists various reliability software, understanding the objective and performing proper methodology are fundamental for the analysis to be meaningful. Most software are generally applicable only at analysis stage where all data have been pre-screened (eg. Time between failures (TBF) were already pre-calculated). In actual scenario, determining accurate TBF requires careful calculation in order to capture the exact operating time. In a simple mean time between failure (MTBF) calculations, MTBF is determined by dividing calendar time with the number of failures, where downtime is considered negligible. In real situation, this approach can be too optimistic since sometimes the downtime can be very long resulted in false long MTBF. Hence, in many situations, the operating time is a more proper measure for MTBF compared to the calendar time.

To address such problems, there is a need to develop a computer assisted analysis program to assist engineer in performing reliability analysis on plant data. This paper aims to present a study on the development of such reliability analysis toolkit. The objectives of the toolkit are:

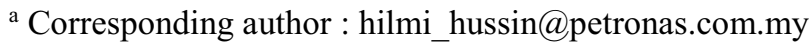

This is an Open Access article distributed under the terms of the Creative Commons Attribution License 2.0, which permits unrestricted use, distribution, and reproduction in any medium, provided the original work is properly cited. 
- to make it easier for engineers to capture relevant data

- to perform exploratory analysis

- to assess data for IID (independent and identically distributed) condition

- to estimate reliability measures

\section{Literature review}

In general, reliability analysis can be done in two stages; exploratory and inferential analysis as shown in Fig. 1. Exploratory data analysis is the process of using statistical tools and techniques to investigate data sets in order to gain insight about the data [1]. Because of this apparent significance, it is recommended to use of exploratory analysis at the beginning of any plant reliability data analysis process [2]. The main outcomes of analysis are the identification of key factors affecting system lifetimes and downtime. Knowing these, management can focus on bad equipment or system to further improve their performance. The purpose of inferential analysis is to determine the best statistical model to represent the data [3]. Two major portions of works involved namely testing for independent and identically distributed (IID) data and fitting into lifetime distribution. For nonrepairable items, the data is assumed IID, and hence can be directly assessed for lifetime distribution analysis (LDA). The data for repairable items, on the other hand, need to be arranged in chronological ordered before they can be tested for IID assumption. The graph of the cumulative number of failures against cumulative operating time between failures can provide a snapshot of how the system performance is heading to. When the inter-arrival time (time between failures) is getting shorter, the plot will tend to concave up signifies that the system is deteriorating. Besides graphical analysis, these conditions can be assessed using analytical trend test, Laplace test, which basically tests whether the process has a monotonic trend or not (stationary) [4].

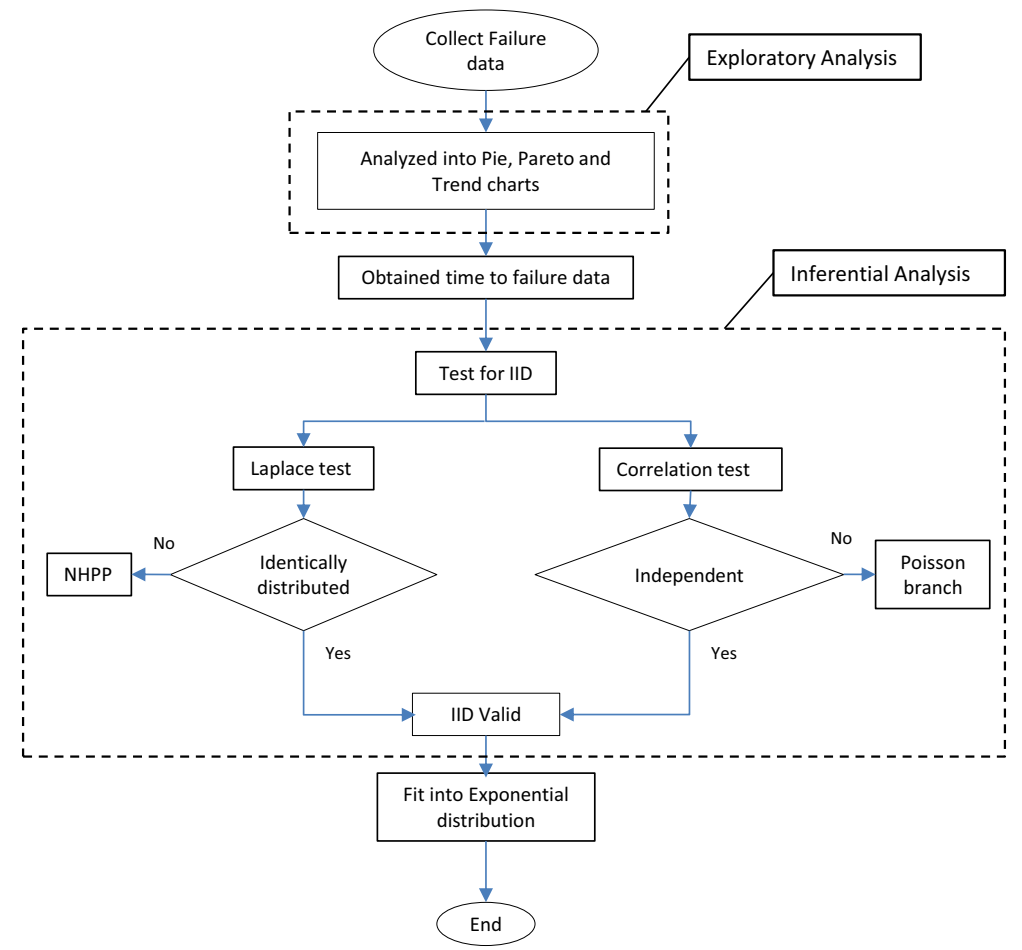

Figure 1. General reliability analysis framework 


\section{Methodology}

The general framework for reliability analysis approach shown in Fig. 1 is used as the basis for toolkit development. The toolkit however did not cover Non-homogeneous Poisson process (NHPP) and Poisson branch testing. The details framework for developing the toolkit is illustrated in Fig. 2. The toolkit is developed using Visual Basics for Applications (VBA) program in Excel 2010 software.

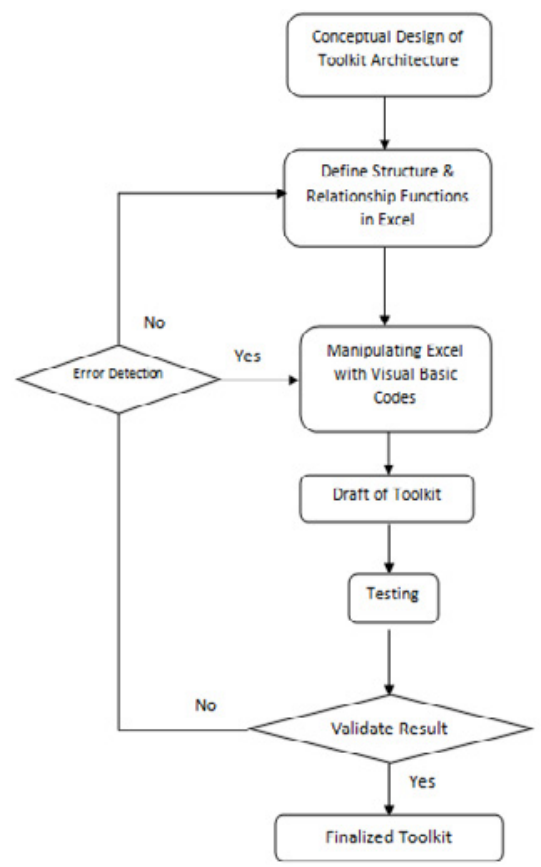

Figure 2. Framework for development of Reliability Analysis Toolkit

\section{Results and discussions}

The resulted user interface screen is shown in Fig.3. Two analysis options are available; exploratory and inferential. Exploratory analysis can be used for non-repairable and repairable system, whereas inferential analysis is mainly for repairable system. For the purpose of demonstration, real plant failure data of an offshore gas compression system are used as input to the Toolkit.

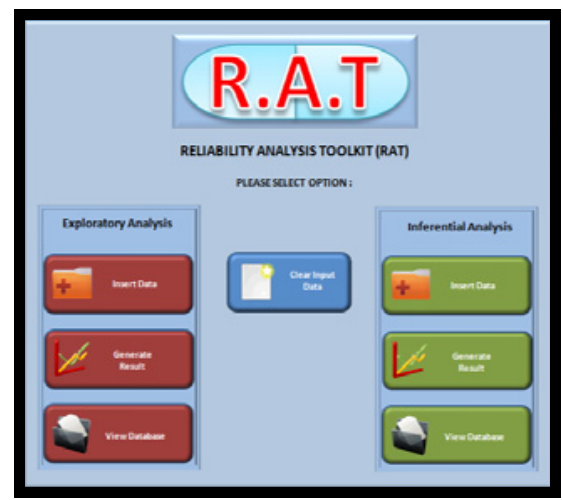

Figure 3. Toolkit interface 


\subsection{Exploratory analysis}

In this analysis, the inputs required are the time and duration of each failure event. The toolkit can perform analysis on the data and the results are presented in graphical forms: Pie; Pareto and Trend charts, which enable management to identify critical system or area for further action or improvement. Example of the Pareto chart for the system based on the input data is illustrated in Fig. 4. The chart indicates that the highest failure is from gas turbine.

Figure 4. Pareto analysis

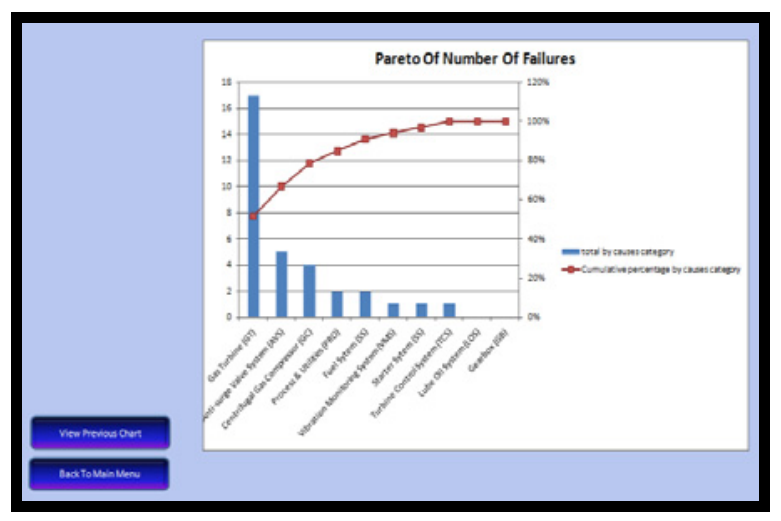

\subsection{Inferential analysis}

The user needs to first identify the exact time and duration of all failure events and associated downtime such as preventive maintenance (PM), standby, turnaround etc. The toolkit will analyze all these data to determine the accurate operating time and time between failures (TBF). This definitely will reduce analysis time as assessor does not need to sort all data in chronological time. The TBF data is then tested for independent and identically distributed (IID) condition based on analytical (Laplace test) and graphical methods. In graphical method, the cumulative number of failures is plotted against the cumulative TBF (operating hours). Fig. 5 depicts such plot for the system which indicates that the data is identically distributed. The plot also implies that the system performance is in a steady state. The toolkit also produces a serial correlation plot for independence test as shown in Fig. 6. There is no pattern observed in the plot indicates that the data are independent.

Finally, the results of Laplace test and estimation of reliability measures, mean time between failures (MTBF) and failure rates, are generated in the toolkit as illustrated in Fig. 7. L value of 1.907 is less than the critical value of 1.95 at $95 \%$ confidence level, hence indicates that there is no monotonic trend in the data.

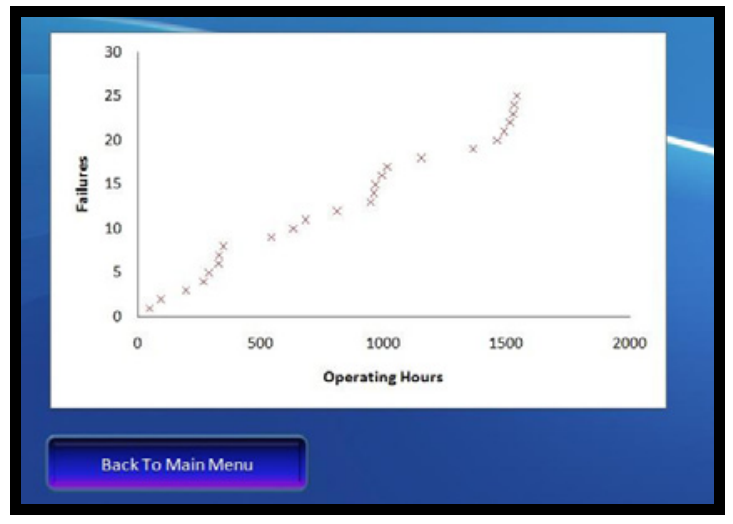

Figure 5. Trend chart 


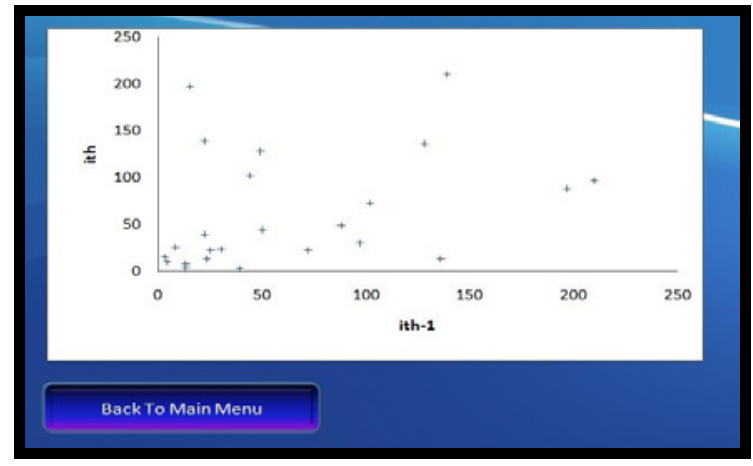

Figure 6. Serial correlation plot

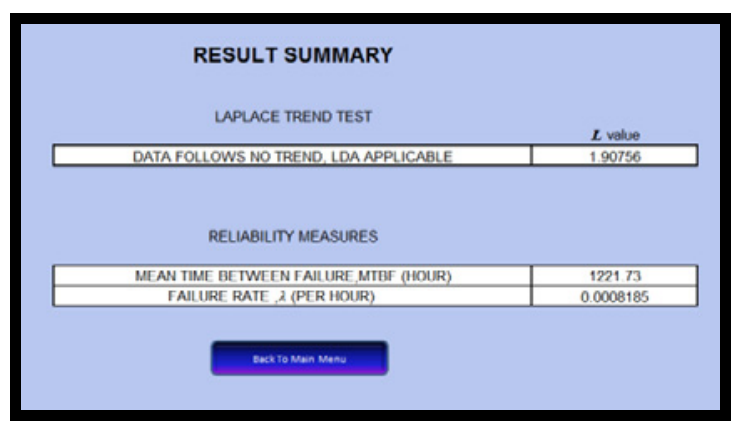

Figure 7. Laplace test and reliability measures estimation results

\section{Conclusions}

In conclusion, the toolkit has been demonstrated capable of facilitating failure data entry, producing exploratory charts (Pie, Pareto and trend charts), testing repairable system data for IID condition and producing reliability measures. Hence, it is can be applied in industry to assist engineer in performing reliability analysis more efficiently.

\section{References}

1. NIST/SEMATECH, e-Handbook of Statistical Methods. (2011). Information on http://www.itl.nist.gov/div898/handbook/apr/section1/apr124.htm

2. M. T. Todinov, Reliability and risk models: setting reliability requirements, John Wiley and Sons, West Sussex, England (2005)

3. T. Fu-rong, J. Zhi-bin and B. Tong-shuo, Reliability Analysis of Repairable Systems Using Stochastic Point Processes, J. Shanghai Jiaotong University (Science), 13(3), 366-369 (2008)

4. D. M. Louit, R. Pascual, and A.K.S. Jardine, A practical procedure for the selection of time-tofailure models based on the assessment of trends in maintenance data, Reliability Eng. \& System Safety, 94 (10), 1618-1628 (2009) 